\title{
ELABORACIÓN DE UN ARTÍCULO CIENTÍFICO DE INVESTIGACIÓN
}

\author{
RESEARCH SCIENTIFIC ARTICLE: KNOW HOW \\ ELENA HENRÍQUEZ FIERRO* y MARIA INÉS ZEPEDA GONZALEZ**
}

\begin{abstract}
RESUMEN
El artículo presenta la forma de redactar correctamente un artículo científico como reporte de una investigación, contempla cada una de las etapas que debe contener para su aprobación. Se sugierela forma de redactar desdeel título hasta la bibliografía, en un lenguaje comprensible y científico. Enfatiza en cómo deben ser presentados los resultados obtenidos para su mejor comprensión de la comunidad científica.
\end{abstract}

Palabras claves: Artículo científico, producción, investigación.

\section{ABSTRACT}

This article introduces ways of writing correctly an article as a research report. It includes every step that has to be taken into account for its approval. The authors provide suggestions of the report writing going from the title to the references, both, in a comprehensive and scientific language. It emphasizes on how the results must be presented for a better understanding of the scientific community.

Keywords: Scientific article, production, research

Recepcionado: 10.05.2004. Aceptado:11.06.2004

\section{INTRODUCCIÓN}

El objetivo del presente artículo es guiar a quienes se inician en la escritura científica, paso a paso en sus etapas, destacando los aspectos más relevantes.

La etapa final de una investigación es comunicar los resultados, de manera que éstos permitan integrar los conocimientos ala práctica profesional, es decir, sebasa en los hallazgos deestudios científicos que deben tener validez, importancia, novedad y utilidad para el quehacer profesional. En relación a las publicaciones científicas, existe una serie de mitos: se cree, por ejemplo, que si una revista es de prestigio, publicar en ella es signo de validez o quesi el autor es prestigioso la publicación será de gran valor; se piensa que si un autor ha publicado con anterioridad un muy buen trabajo, ello es garantía futura de val idez científica o que estálibre de prejuicios y de sesgos. Esto no es tan cierto, en el trabajo investigativo y sus resultados pesan muchos otros elementos que se presentan en este artículo.

Esta etapa final es similar a la etapa inicial en el grado de dificultad que involucra. Para interpretar y comunicar los resultados de un estudio se requiere experiencia, conocimiento de la estadística y capacidad de análisis para realizar los comentarios pertinentes relacionándolo con otros hallazgos de investigacionessimilares, incluyendo creatividad del autor o autores, compenetración intelectual, razonamiento lógico y sensibilidad frentea lasinterpretaciones que se pueden dar. 
Generalmente, finalizado un estudio setie nen sólo números, que es el resultado de los datos obtenidos pero que no tienen un significado claro si no son interpretados por el autor; y es el investigador el quetienequeimprimirle esta connotación, es decir, buscar el significado y transcendencia de éstos. Se deben considerar los objetivos o hipótesis planteados en el proyecto con el análisis de los resultados, existiendo una clara concordancia entre ellos. Ideal mente se debe tener una teoría quesustentela interpretación de los resultados. Éstos deben definirse con claridad y de acuerdo a quienes serán los usuarios o receptores de los hallazgos.

Existen principalmente dos contextos a los cuales pueden presentarse:

\section{a) Contexto académico \\ b) Contexto no académico}

En el primer caso los resultados se presentan a un grupo de profesores-investigadores, alumnos de una institución de educación superior, lectores con niveles educativos el evados o miembros de una agencia de investigación o con perfil similar.

En el contexto no académico, los resultados pueden ser presentados con fines comerciales o a público en general.

La diferencia radica en la naturaleza y extensión del reporte; sin dejar demencionar el objetivo, dónde se realizó el estudio, cuándo, aspectos metodológicos, resultados y conclusiones que se obtuvieron.

En esta etapa es fundamental que los estadísticos el egidos para el procedimiento analítico sean claros y que se hayan aplicado correctamente para el nivel de medición de los datos y analizados en relación a los objetivos del estudio.

En el caso de las variables estudiadas, éstas deben presentarse en grupos lógicos. Una vez que se tiene claridad de lo que se va a comunicar, generalmente surge el artículo para ser presentado en una revista.

Las etapas que debe incluir el informe de un artículo científicos son:
I. Introducción
II. M etodología
III. Resultados
IV. Discusión y conclusión
V. Bibliografía

\section{DEL TÍTULO}

Se inicia con el nombre del artículo, que debe claramente indicar el contenido del estudio, es decir, el problema de investigación y variables principales; debe ser claro, fácil de entender, conciso no más de 15 palabras, debecontener las palabras claves o descriptores del estudio, no utilizar abreviaturas, usando tiempo afirmativo. No debe ser partido, es decir, no separar en frases, usar un lenguaje senciIlo, términos claros y directos, no contener sobreexplicaciones.

\section{DE LOSAUTORES}

Destacar si es autoría múltiple o individual, justificada, responsable; los nombres deben ser completos y sin iniciales. Es necesario poner al margen instituciones de trabajo, sin incluir grados académicos o posiciones jerárquicas, además de la dirección postal o electrónica del investigador encargado de la correspondencia.

\section{DEL RESUMEN}

Este breve contenido debe permitir identificar el aspecto básico del informe de forma rápida y exacta, fácil de entender, conteniendo el objetivo o hipótesis en el primer párrafo. La metodología está presente en el segundo párrafo, allí se describeclaramenteel diseño. En el tercer párrafo se deben presentar los resultados principales y, en el cuarto, las conclusiones. La extensión del resumen no debe ir más allá de 250 palabras.

Se sugiere además que los resultados sean con valores numéricos, ya sea en números, ta- 
sas, porcentajes o proporciones; no usar abreviaturas, ni siglas. El texto no debe incluir tablas, gráficos ni figuras. En resumen, debe ser autoexplicativo.

\section{INTRODUCCIÓN}

En ésta se presenta claramenteel quéy el porqué de la investigación, incluye el planteamiento del problema, objetivo y preguntas de la investigación, así como la justificación del problema, el contexto general deinvestigación, cómo y dónde se realizó, las variables de la investigación y sus definiciones, así como las limitaciones de ésta. Se puede incluir el marco de referencia o revisión de la literatura.

D ebe captar la atención del lector desdeel primer párrafo introductorio, invitando einteresando al lector a seguir leyendo el documento, con un estilo directo sin dejar espacio a otras interpretaciones.

El tema general se presenta prontamente, para luego pasar al problema deinvestigación, que es el fenómeno específico de interés que debe ser actual, susceptible de observación y de medición, es decir, factible de investigar.

Debeexistir una amplia revisión bibliográfica de lo que se sabe actualmente en función delo publicado sobreel problema, la quedebe ser relevante y reflejar la información sobre antecedentes del problema necesario para apoyar la justificación del estudio. Las referencias citadas en el texto deben estar bien documentadas y actualizadas.

\section{METODOLOGÍA}

\section{a) Diseño}

En este capítulo es importante que el diseño haya sido el apropiado para el objetivo de estudio. Éste debe describirse suficientemente, caracterizando la dimensión de la intervención del Investigador, es decir, cómo él manipuló la/las variables independientes en función de los objetivos que se planteó.
Además debe explicar la dimensión temporal, como el momento y número de veces que fueron necesarios para la recolección de la información.

Debe estar presente la unidad de análisis, con casos, serie decasos, muestra o población total, indicando además el nivel de análisis de la investigación (correlación, causalidad o inferencia y no análisis).

Es de importancia señalar si el diseño corresponde al paradigma epistemológicometodológico (cuantitativo-cualitativo) con los datosquesehan obtenido, respal dados por el nivel de conocimiento disponible y actualizados sobre el problema de investigación.

Finalmentees conveniente recordar queel diseño garantizaun grado de control suficiente, pues contribuye así a la validez interna del estudio. Se debe presentar el criterio de exclusión einclusión.

Resumiendo el diseño debe contener el escenario en que se desarrolló la investigación, el o los sujetos, el tamaño muestral, bajo qué condiciones se trabajó, los métodos de recolección de la muestra y cómo fueron analizados los datos.

\section{b) Población y muestra}

Debe estar identificado y descrito con claridad el segmento de la población total con la que se trabajó (población diana), luego la población accesibleal estudio y explicar si seutilizó un muestreo aleatorio probalístico o un muestreo no probalístico.

En caso de muestreo al eatorio, se debe explicar el procedimiento: al eatorio simple, aleatorio estratificado, al eatorio por conglomerado o aleatorio sistemático.

Si en el muestreo seutilizó el procedimiento no aleatorio, debe explicarse el muestreo de conveniencia, de cuota o intencional, es decir, al tamaño de la muestra se informa a la luz del objetivo del estudio, del diseño, del método de muestreo y del análisis estadístico de los datos. 
Resumiendo, la muestra nos debe indicar cuán representativa es la población diana a la cual selegeneralizan los resultados, por lo que la muestra debe ser suficiente para garantizar la validez externa del estudio .

En caso de grupos de estudio y control, debe estipularse con claridad el método de selección y asignación de sujeto.

\section{c) Consideraciones éticas}

Debe estar descrito cómo se obtuvo el consentimiento informado y la constancia de la revisión de la investigación por un consejo 0 comitédeética dela institución patrocinante, describiendo los riesgos potenciales de los sujetos participantes del estudio, si lo amerita.

La constancia del anonimato y confidencialidad de los participantes del estudio es fundamental.

Además, debe quedar estipulada la dirección postal o electrónica de un miembro del equipo de investigación, para responder alguna consulta referente al consentimiento informado.

\section{RESULTADOS}

Es lo más importante del informe, no dede contener comentarios, ni juicios de valor, ni justificaciones.

Los resultados deben presentarse en el orden que fueron planteados los objetivos, de lo más importante a lo menos significativo.

En la presentación adquiere más importancia el texto que los gráficos y tablas, debiendo ser claro, conciso, preciso y con una secuencia lógica.

\section{a) Recolección de los datos}

Mencionar la razón fundamental de la elección del instrumento utilizado, describiendo su validez y confiabilidad.
Se debe describir claramente los pasos en el procedimiento de recolección de los datos.

\section{b) Análisis de los datos}

Debereflejar quelos procedimientos estadísticos utilizados fueron los correctos para el nivel de medición de los datos y que se analizan en relación con los objetivos de estudio. Si éstetienehipótesis, debequedar claramente estipulado la aprobación o rechazo de ella.

El análisis estadístico debe considerar el nivel de medida para cada una de las variables: nominal, ordinal o intervalo.

Si la investigación contempló grupos de estudio y de control, éstos deben ser comparados, indicando con precisión la duración del estudio (seguimiento) para ambos.

Sedebe considerar si los datos fueron analizados por técnicas cualitativas, cuantitativas o ambas y señalar los pasos que se siguieron para validar los resultados.

\section{c) Presentación de los datos}

Se debefocalizar hacia los hallazgos pertinentes y respondiendo a la pregunta de investigación y/o a la prueba de hipótesis.

Los datos deben presentarse en forma específica, sin comentarios ni argumentos. El texto comanda la presentación en forma clara, precisa y concisa.

Los resultados se presentan en forma ordenada siguiendo el orden de los objetivos / hipótesis.

Seinician con los hallazgosmásimportantes, dejando las asociaciones negativas para el final de la sección, considerando el riesgo relativo y del intervalo de confianza.

Se deben utilizar términos estadísticos en su relato (significación, aleatorio, muestra, correlación, regresión, etc.).

Las tablas y gráficos deben ser simples, autoexplicativos y autosuficientes, incluyendo datos numéricos. 
Las tablas y los gráficos complementan el texto y ayudan a una comprensión rápida y exacta delos resultados, clarifican la información, ponen énfasis en los datos más significativos, establecen relaciones y resumen el material de los hallazgos.

Las tablas se utilizan cuando los datos tienen resultados exactos y con decimales con encabezamiento de filas y columnas; nunca deben presentarse cortadas. No es necesario la fuente de la tabla ya que es producto del trabajo que se realizó.

Losgráficosseusan cuando sequiere mostrar el comportamiento de una variableen un período detiempo. Presentando con más claridad que una tabla, una tendencia. Se recomienda la enumeración de las tablas y gráficos para facilitar su consulta.

\section{DISCUSIÓN Y CONCLUSIONES}

El estilo de la discusión debe ser argumentativo, haciendo uso juicioso de la polémica y debate por parte del autor, para convencer al lector que los resultados tienen validez interna y externa. Esto contrasta con el estilo descriptivo y narrativo dela introducción, material y método y resultado.

Sesugierediscutir en primera instancia los resultados propiosy losmás importantes, luego se comparan los resultados propios con los de otros estudios similares publicados, de acuerdo a la revisión bibliográfica; es factible incluir las implicaciones teóricas y prácticas, recomendando sobre posibles futuras investigaciones relativas al tema.

Las conclusiones deben ser presentadas claramentecomo respuesta a la interroganteque originó el estudio y a los objetivos planteados, por lo tanto debe haber tantas conclusiones como objetivos.

Esconveniente dejar en claro las limitaciones que el estudio presentó y la forma como pudieron afectar las conclusiones.

Resumiendo, una buena discusión no comenta todos los resultados, no los repite de capítulos anteriores, sin confundir hechos u opiniones, sin hacer conjeturas.

Además, no generaliza, no infiere, ni extrapola en forma injustificada y no plantea comparaciones teóricas sin fundamento.

\section{BIBLIOGRAFÍA}

Fundamental es tener las referencias adecuadas; en este sentido los descriptores del título del artículo deben coincidir con los descriptores de las referencias; éstas deben ser actualizadas y corresponden a un $50 \%$ de los últimos 5 años, el resto puede ser de no más de 5 años antes y por excepción se aceptan referencias de publicaciones "clásicas" de más años.

Deben presentarse no menos de 30 referencias en un artículo científico de una investigación, siendo el $50 \%$ de éstas publicaciones de tipo primario.

Cada revista tiene exigencias particulares, pero en general las referencias deben incluir: autor, título, lugar de publicación, editorial y año, en caso de libro; autor, título, nombrede revista, volumen y páginas, en caso de revista.

\section{REFERENCIASBIBLIOGRÁFICAS}

BOBENRIETH ASTETE, M .A. (1998). “Escritura y lectura crítica de artículos científicos". En: Burgos Rodríguez R. Metodología de investigación y escritura científica en clínica. ParteIV. Granada. Escuela Salud Pública.

BOBEN RIETH ASTETE, M .A. (2000). “M itos y realidades en torno a la publicación científica". M edicina Clínica, 114: 339-341.

BOBEN RIETH , M . (2000). Curso actualización en escritura científica. Nivel avanzado, pp. 7-22.

BOBEN RIETH , M . (2000). Curso actualización en escritura científica. EASP. Granada, pp. 2-29.

CANALES, F. et al. (1994). M etodología de la Investigación OPS - OMS, $2^{\text {da }}$ edición.

HERNANDEZ, R. et al. (1998). Metodología de la investigación Ed. M c. Graw-Hill, $2^{\text {da }}$ edición.

POLIT, D. et al. (1998). Investigación científica en ciencias de la salud. Ed. M c. Graw-Hill, $4^{\text {ta }}$ edición. 
